\title{
Analysis of Factors Affecting the Outcome of Flap Reconstruction for Pressure Ulcers
}

\author{
Donghwan Lee (D), Min Ji Kim (D), Hyung Min Hahn (D) \\ Department of Plastic and Reconstructive Surgery, Ajou University School of Medicine, Suwon, Korea
}

\begin{abstract}
Background: Pressure ulcers are a common and challenging problem affecting bed-ridden patients. Flap operations are considered a surgical option for treating pressure sores despite their high rate of complications and recurrence. Hypothesizing that certain factors would affect postoperative outcomes for pressure sore patients, we analyzed the factors and outcomes.

Methods: We collected cases retrospectively from January 2010 to January 2018. The subjects were 95 patients who had undergone flap operation in our institution. Patient demographics, wound characteristics, and outcomes including relapses, flap complications, reoperation, and newly developed sores were analyzed. Logistic regression analysis was used to identify the association between variables and surgical outcomes.

Results: Patients who had larger area of defects after debridement had more flap complications $(P=0.01)$ and were more likely to undergo reoperation $(P=0.03)$. Smokers $(P=0.04)$ and patients whose wound cultures were positive $(P=0.04)$ had more flap complications. Patients who had paralysis were more likely to require reoperation $(P=0.02)$ and to develop new sores $(P=0.02)$.

Conclusion: We identified factors affecting postoperative outcomes after flap operation to cover pressure sores. As a more comprehensive preoperative risk assessment on flap reconstruction for pressure sores can improve outcomes, these indicators should be carefully considered when determining surgical options.
\end{abstract}

Keywords: Pressure ulcer; Surgical flap; Reconstruction

\section{Introduction}

Pressure on any part of the body can damage the skin and underlying tissues, resulting in a pressure ulcer. Many hospitalized and long-term care patients suffer from pressure ulcers [1]. Considering the rising healthcare expenses and the burden on society in general [2-4], more efforts should be made to prevent the development of pressure ulcers in high-risk patients.

Flap operations are the typical treatment used to cover pressure ulcer wounds. Flaps are considered a good option because they provide sufficient volume and reliable vascular supply [5]. Since flap surgery has become the preferred treatment, few physicians consider the alternatives of primary closure or skin grafts.

However, complications after surgery is always a concern [6,7]. Complications may arise because of the condition of each patient, or due to the nature of a flap operation [8]. In addition, most patients with pressure ulcers are not capable of voluntary movement and suffer from general weakness [9]. These limitations mean that recurrence is common, and when ulcers recur, the range of options for coverage is reduced [10]. This compounds the need to reduce recurrence and complications when performing surgery, thereby decreasing the likelihood that the patient would need a reoperation. Several studies have investigated the risk factors of flap surgery; however, their popu-

\section{Original Article}

Received: August 24, 2020

Revised: November 7, 2020

Accepted: November 8, 2020

\section{Corresponding author: \\ Hyung Min Hahn, M.D.}

Department of Plastic and Reconstructive Surgery, Ajou University School of Medicine, 206 World cup-ro, Yeongtong-gu, Suwon 16499, Korea

Tel: +82-31-219-5241

Fax: +82-31-219-5164

E-mail: newcetizen@naver.com

This is an Open Access article distributed under the terms of the Creative Commons Attribution Non-Commercial License (https://creativecommons.org/licenses/by-nc/4.0/) which permits unrestricted non-commercial use, distribution, and reproduction in any medium, provided the original work is properly cited.

(C) 2021 Korean Wound Management Society 
lations were limited to spinal cord injury patients, and the reported complication rates varied widely. Further, the previous studies evaluated the severity of recurrence or complications [10-13] insufficiently.

Postoperative outcomes must be anticipated when performing surgical treatment to cure or improve pressure ulcers [11]. We predicted that certain risk factors would correlate to different postoperative outcomes, such as relapse after a successful operation, complications after an operation, postoperative wounds that require reoperation, and newly developed pressure sores. In this study, we aimed to investigate the relationship between perioperative variables and postoperative outcomes to identify patients with higher risk of poor outcomes.

\section{Methods}

Medical charts of patients who underwent flap operations for pressure ulcers between January 2010 and February 2018 were reviewed retrospectively. Among the patients admitted to our department, we selected patients with an L89 diagnosis and subtypes associated with a decubitus ulcer, according to the ICD-10 (10th revision of the International Statistical Classification of Diseases and Related Health Problems) disease code. Only patients who had a flap surgery to cover the pressure sore were included. We excluded patients who had simple debridement, incision and drainage, and bursectomy, and those with pressure sores treated with conservative wound care. In addition, while patients whose pressure ulcer wounds underwent primary repair or skin grafts were also excluded, patients who underwent primary repair or skin graft at the donor site were included. This study was performed in accordance with the principles of the Declaration of Helsinki. The study and all its protocols were approved by the Institutional Review Board of Ajou Medical Center (IRB No. AJIRB-MED-MDB-17-254). The need for informed consent was waived by the institutional review board of our hospital due to the retrospective design of the study.

The following demographic information on the patients were collected: age, sex, body mass index (BMI), weight, comorbidities (diabetes and end-stage renal disease [ESRD]), smoking, paralysis (previously diagnosed paraplegia, hemiplegia, and quadriplegia), and serum albumin and hemoglobin level. Patients' activity levels were evaluated by the Eastern Cooperative Oncology Group (ECOG) performance status and Braden scale score. In terms of wound characteristics, wound area size, culture results, and location were noted. The wound area was assessed from the measurements for designing the flaps for during reconstruction. The culture tests were routinely obtained on the first day of hospitalization.

Postoperative outcomes included relapse, complications, reoperation, and a newly developed sore at other sites. Surgical complications were defined as all surgery-related problems, including wound dehiscence, infection, necrosis, hematoma, and seroma. A relapse was defined as a pressure sore reoccurring during follow-up in the same area after flap reconstruction. Reoperation was defined as the surgical site being reoperated upon, owing to complications or relapse. Reoperations included debridement, primary closure, and flap surgery, and its necessity was determined at the discretion of the surgeon. If the surgeon decided that the wound could not heal with conservative treatment, it was given a reoperation. Pressure sores occurring in other areas during follow-up were also considered.

R language version 3.3.3 (R Foundation for Statistical Computing, Vienna, Austria) and T\&F program ver. 2.2 (Yoo)in BioSoft, Goyang, Korea) were used for all statistical analyses. Data were expressed as mean \pm standard deviation (SD) for continuous variables. For categorical variables, data were expressed as sample number and percentage, number (\%). When continuous variables were normally distributed, a mean difference test between two subgroups of outcomes was performed using the Student t-test. For non-normally distributed variables, the Mann-Whitney U test was used. For categorical variables, chi-square test or Fisher exact test were performed to test the hypothesis of association between outcome and other variables as appropriate, using contingency tables. For analyzing the combined effect on the outcome of more than two variables, multivariable linear regression analysis was performed using backward stepwise procedure. To analyze the effect of each clinical measurement on the binary outcomes of relapse, flap complication, reoperation, or new sore development, binary logistic regression analysis was performed. For analyzing the combined effect of more than two variables on the binary outcome, multivariable logistic regression analysis was performed using backward stepwise procedure.

\section{Results}

During the period included in the study, a total of 757 patients had surgery for pressure ulcers in our department. The 384 patients who had only simple debridement, incision and drainage, and bursectomy were excluded, and 278 who had 
Table 1. Characteristics of demographic and clinical variables

\begin{tabular}{|c|c|c|}
\hline Variable & Subgroup & №. (\%) \\
\hline Age, mean $\pm S D(y r)$ & & $53.95 \pm 9.44$ \\
\hline \multirow[t]{2}{*}{ Sex } & Female & $40(42.1)$ \\
\hline & Male & $55(57.9)$ \\
\hline \multirow[t]{4}{*}{ BMI $\left(\mathrm{kg} / \mathrm{m}^{2}\right)$} & $<20$ & $29(30.5)$ \\
\hline & 20 to $<25$ & $47(49.5)$ \\
\hline & 25 to $<30$ & $13(13.7)$ \\
\hline & $\geq 30$ & $6(6.3)$ \\
\hline Weight, mean \pm SD (kg) & & $60.81 \pm 6.87$ \\
\hline \multirow[t]{2}{*}{ Diabetes } & № & $69(72.6)$ \\
\hline & Yes & $26(27.4)$ \\
\hline \multirow[t]{2}{*}{ ESRD } & № & 89 (93.7) \\
\hline & Yes & $6(6.3)$ \\
\hline \multirow[t]{2}{*}{ Smoker } & № & $79(83.2)$ \\
\hline & Yes & $16(16.8)$ \\
\hline \multirow[t]{2}{*}{ Paralysis } & № & $57(60.0)$ \\
\hline & Yes & $38(40.0)$ \\
\hline \multirow[t]{4}{*}{ ECOG performance status } & 1 & $13(13.7)$ \\
\hline & 2 & $15(15.8)$ \\
\hline & 3 & $19(20.0)$ \\
\hline & 4 & $48(50.5)$ \\
\hline \multirow[t]{4}{*}{ Albumin, mean $\pm S D(g / d L)$} & & $3.55 \pm 0.32$ \\
\hline & $<3.0$ & $22(23.2)$ \\
\hline & 3.0 to $<3.5$ & $20(21.1)$ \\
\hline & 3.5 to $<5.2$ & $53(55.8)$ \\
\hline \multirow[t]{4}{*}{ Hemoglobin, mean $\pm S D(g / d L)$} & & $11.31 \pm 0.90$ \\
\hline & $<10.7$ & $39(41.1)$ \\
\hline & $10.7-15.3$ & $55(57.9)$ \\
\hline & $>15.3$ & $1(1.1)$ \\
\hline \multirow[t]{6}{*}{ Braden scale, mean $\pm S D$} & & $13.80 \pm 1.49$ \\
\hline & Severe risk & $6(6.3)$ \\
\hline & High risk & $20(21.1)$ \\
\hline & Moderate risk & $33(34.7)$ \\
\hline & Mild risk & $28(29.5)$ \\
\hline & No risk & $8(8.4)$ \\
\hline Area, mean $\pm S D\left(\mathrm{~cm}^{2}\right)$ & & $29.09 \pm 18.80$ \\
\hline \multirow[t]{2}{*}{ Culture } & Negative & $55(57.9)$ \\
\hline & Positive & $40(42.1)$ \\
\hline \multirow[t]{3}{*}{ Site } & Ischial area & $22(23.2)$ \\
\hline & Sacral area & $59(62.1)$ \\
\hline & Trochanter & $14(14.7)$ \\
\hline
\end{tabular}

(Continued to the next)
Table 1. Continued

\begin{tabular}{llc}
\hline Variable & Subgroup & №. (\%) \\
\hline Relapse & No & $75(78.9)$ \\
& Yes & $20(21.1)$ \\
Flap complication & No & $78(82.1)$ \\
Reoperation & Yes & $17(17.9)$ \\
Sore newly developed on other sites & No & $68(71.6)$ \\
& Yes & $27(28.4)$ \\
& Yes & $88(92.6)$ \\
\hline
\end{tabular}

BMI, body mass index; ESRD, end-stage renal disease; ECOG, Eastern Cooperative Oncology Group.

primary repair or skin graft instead of a flap surgery were also excluded. The remaining 95 patients who had a flap operation for pressure ulcers were ultimately included in the retrospective case-control study.

The patient demographics are summarized in Table 1. The mean age was 53.95 years. There were 55 male patients, accounting for $57.9 \%$ of the sample. Twenty-nine patients (30.5\%) had a BMI of $20 \mathrm{~kg} / \mathrm{m}^{2}$ or less, thirteen patients (13.7\%) recorded a BMI between $25-30 \mathrm{~kg} / \mathrm{m}^{2}$, and six patients $(6.3 \%)$ had a BMI over $30 \mathrm{~kg} / \mathrm{m}^{2}$. Of the comorbidities, 26 patients (27.4\%) had diabetes and 6 (6.3\%) had ESRD. There were 16 smokers (16.8\%) in this study. Thirty-eight patients (40\%) had paralysis, and forty-eight patients (50.5\%) had an ECOG score of 4. Patients whose levels of albumin $(\leq 3.0 \mathrm{~g} / \mathrm{dL})$ and hemoglobin $(<10.7 \mathrm{~g} / \mathrm{dL})$ were below normal amounted to $22(23.2 \%)$ and 39 (41.1\%) patients, respectively. The Braden scale scores were 6 (6.3\%), 20 (21.1\%), and 33 (34.7\%) for the severe, high, and moderate risk groups, respectively.

The average defect size of the wounds was $29.09 \mathrm{~cm}^{2}( \pm 18.80)$ culture result was positive in 40 patients (42.1\%) and the location of the sores were in the ischial area for 22 patients (23.2\%), in the sacral area for 59 patients (62.1\%), and in the trochanter area for 14 patients (14.7\%). The recurrence rate of pressure ulcers in the same area after flap reconstruction was $20.1 \%$. Flap complications occurred in 17 patients (17.9\%). There were 27 patients $(28.4 \%)$ who had relapses or flap complications to the extent that they had to receive reoperations, and 7 patients (7.4\%) developed pressure sore at other sites during the follow-up.

Table 2 shows the effects of clinical variables on flap complication occurrence. Sore size and smoking positively correlated with flap complications, while age, Braden scale score, albu- 
Lee $\mathrm{D}$ et al.

Outcome of flap reconstruction of sore

Table 2. Analysis of effects of clinical variables on flap complication occurrence

\begin{tabular}{|c|c|c|c|c|c|c|c|}
\hline \multirow{2}{*}{ Variable } & \multirow{2}{*}{ Subgroup } & \multicolumn{2}{|c|}{ Flap complications, №. (\%) } & \multicolumn{2}{|c|}{ Unadjusted analysis } & \multicolumn{2}{|c|}{ Adjusted analysis } \\
\hline & & No & Yes & OR (95\% Cls) & P-value & OR (95\% Cls) & P-value \\
\hline Age, mean $\pm S D$ (yr) & & $54.51 \pm 2.14$ & $51.35 \pm 4.68$ & $1.009(0.981-1.037)$ & 0.53 & $1.018(0.982-1.055)$ & 0.54 \\
\hline \multirow[t]{3}{*}{ Sex } & & & & & 0.16 & & 0.32 \\
\hline & Female & $31(39.7)$ & $9(52.9)$ & 1 & & 1 & \\
\hline & Male & $47(60.3)$ & $8(47.1)$ & $0.321(0.066-1.567)$ & & $0.586(0.204-1.684)$ & \\
\hline \multirow[t]{5}{*}{ ECOG performance status } & & & & & 0.41 & & 0.35 \\
\hline & 1 & $10(12.8)$ & $3(17.6)$ & 1 & & 1 & \\
\hline & 2 & $13(16.7)$ & $2(11.8)$ & $0.991(0.231-4.247)$ & & $0.513(0.072-3.677)$ & \\
\hline & 3 & $18(23.1)$ & $1(5.9)$ & $1.932(0.377-9.900)$ & & $0.185(0.017-2.024)$ & \\
\hline & 4 & $37(47.4)$ & $11(64.7)$ & $5.351(0.640-44.725)$ & & $0.991(0.231-4.247)$ & \\
\hline \multirow[t]{3}{*}{ Paralysis } & & & & & 0.83 & & 0.51 \\
\hline & No & $48(61.5)$ & $9(52.9)$ & 1 & & 1 & \\
\hline & Yes & $30(38.5)$ & $8(47.1)$ & $1.079(0.541-2.153)$ & & $1.422(0.495-4.089)$ & \\
\hline \multirow[t]{5}{*}{$\mathrm{BMI}\left(\mathrm{kg} / \mathrm{m}^{2}\right)$} & & & & & 0.33 & & 0.25 \\
\hline & $<20$ & $24(30.8)$ & $5(29.4)$ & $4.800(0.741-31.081)$ & & $1.190(0.340-4.173)$ & \\
\hline & 20 to $<25$ & $40(51.3)$ & $7(41.2)$ & 1 & & 1 & \\
\hline & 25 to $<30$ & $11(14.1)$ & $2(11.8)$ & $5.714(0.954-34.242)$ & & $1.039(0.188-5.729)$ & \\
\hline & $\geq 30$ & $3(3.8)$ & $3(17.6)$ & $5.500(0.611-49.535)$ & & $5.714(0.954-34.243)$ & \\
\hline Weight, mean $\pm S D(k g)$ & & $60.47 \pm 1.59$ & $62.38 \pm 3.11$ & $0.990(0.953-1.028)$ & 0.60 & $1.069(0.948-1.205)$ & 0.61 \\
\hline \multirow[t]{3}{*}{ Diabetes } & & & & & 0.82 & & 1.00 \\
\hline & No & $57(73.1)$ & $12(70.6)$ & 1 & & 1 & \\
\hline & Yes & $21(26.9)$ & $5(29.4)$ & $1.198(0.244-5.891)$ & & $1.131(0.356-3.597)$ & \\
\hline \multirow[t]{3}{*}{ ESRD } & & & & & 0.69 & & 1.00 \\
\hline & № & $73(93.6)$ & $16(94.1)$ & 1 & & 1 & \\
\hline & Yes & $5(6.4)$ & $1(5.9)$ & $0.564(0.035-9.067)$ & & $0.912(0.100-8.353)$ & \\
\hline \multirow[t]{3}{*}{ Smoker } & & & & & $0.02^{\mathrm{a})}$ & & $0.04^{\text {a) }}$ \\
\hline & No & $68(87.2)$ & $11(64.7)$ & 1 & & 1 & \\
\hline & Yes & $10(12.8)$ & $6(35.3)$ & 7.178 (1.459-35.309) & & 3.709 (1.122-12.264) & \\
\hline \multirow[t]{3}{*}{ Culture } & & & & & 0.07 & & $0.04^{\mathrm{a})}$ \\
\hline & Negative & $49(62.8)$ & $6(35.3)$ & 1 & & 1 & \\
\hline & Positive & $29(37.2)$ & $11(64.7)$ & $3.570(0.897-14.205)$ & & $3.098(1.036-9.265)$ & \\
\hline \multirow[t]{4}{*}{ Site } & & & & & 0.91 & & 0.86 \\
\hline & Ischial area & $19(24.4)$ & $3(17.6)$ & 1 & & 1 & \\
\hline & Sacral area & $48(61.5)$ & $11(64.7)$ & $1.525(0.228-10.209)$ & & $1.451(0.364-5.785)$ & \\
\hline & Trochanter & $11(14.1)$ & $3(17.6)$ & $1.203(0.265-5.461)$ & & $1.727(0.296-10.082)$ & \\
\hline Area, mean $\pm S D\left(\mathrm{~cm}^{2}\right)$ & & $23.68 \pm 3.35$ & $53.96 \pm 13.91$ & $0.983(0.969-0.996)$ & $0.01^{\mathrm{b})}$ & $0.978(0.957-0.999)$ & $0.01^{\text {b) }}$ \\
\hline \multirow[t]{6}{*}{ Braden scale } & & & & & 1.00 & & 0.81 \\
\hline & Severe risk & $6(7.7)$ & 0 & 1 & & 1 & \\
\hline & High risk & $16(20.5)$ & $4(23.5)$ & - & & $3.545(0.166-75.580)$ & \\
\hline & Moderate risk & $27(34.6)$ & $6(35.3)$ & $1.000(0.086-11.588)$ & & $3.679(0.185-73.058)$ & \\
\hline & Mild risk & $23(29.5)$ & $5(29.4)$ & $0.929(0.089-9.687)$ & & $3.043(0.148-62.509)$ & \\
\hline & No risk & $6(7.7)$ & $2(11.8)$ & $1.150(0.105-12.616)$ & & $4.333(0.142-132.320)$ & \\
\hline \multirow[t]{4}{*}{ Albumin (g/dL) } & & & & & 0.52 & & 0.63 \\
\hline & $<3.0$ & $19(24.4)$ & $3(17.6)$ & $0.577(0.113-2.934)$ & & $0.772(0.188-3.172)$ & \\
\hline & 3.0 to $<3.5$ & $15(19.2)$ & $5(29.4)$ & $2.225(0.661-7.482)$ & & $1.630(0.471-5.634)$ & \\
\hline & 3.5 to $<5.2$ & $44(56.4)$ & $9(52.9)$ & 1 & & 1 & \\
\hline \multirow[t]{3}{*}{ Hemoglobin (g/dL) } & & & & & 0.20 & & 0.27 \\
\hline & $\geq 10.7$ & $48(61.5)$ & $8(47.1)$ & 1 & & 1 & \\
\hline & $<10.7$ & $30(38.5)$ & $9(52.9)$ & $2.225(0.661-7.482)$ & & $1.800(0.626-5.175)$ & \\
\hline
\end{tabular}

Odds ratio (OR) and $95 \%$ confidence intervals (Cls) computed in the subsample of flap complication; No vs. yes, P-value computed using Student t-test and Mann-Whitney $\mathrm{U}$ test in continuous variables; $P$-value computed using chi-square test and Fisher exact test in categorical variables, age, weight, and area were continuous variables and expressed mean \pm SD. ECOG, eastern cooperative oncology group; BMI, body mass index; ESRD, end-stage renal disease.

${ }^{\text {a) }} \mathrm{P}<0.05 ;{ }^{\mathrm{b})} \mathrm{P}<0.01$. 
Lee $\mathrm{D}$ et al.

Outcome of flap reconstruction of sore

Table 3. Analysis of effects of clinical variables on reoperation

\begin{tabular}{|c|c|c|c|c|c|c|c|}
\hline \multirow{2}{*}{ Variable } & \multirow{2}{*}{ Subgroup } & \multicolumn{2}{|c|}{ Reoperation, №. (\%) } & \multicolumn{2}{|c|}{ Unadjusted analysis } & \multicolumn{2}{|c|}{ Adjusted analysis } \\
\hline & & No & Yes & OR (95\% Cls) & P-value & OR (95\% Cls) & P-value \\
\hline Age, mean $\pm S D$ (yr) & & $55.56 \pm 2.38$ & $49.89 \pm 3.20$ & $1.016(0.992-1.041)$ & 0.19 & $1.012(0.975-1.050)$ & 0.19 \\
\hline \multirow[t]{3}{*}{ Sex } & & & & & 0.75 & & 0.87 \\
\hline & Female & $29(42.6)$ & $11(40.7)$ & 1 & & 1 & \\
\hline & Male & $39(57.4)$ & $16(59.3)$ & $0.749(0.123-4.547)$ & & $1.082(0.437-2.675)$ & \\
\hline \multirow[t]{5}{*}{ ECOG performance status } & & & & & 0.64 & & 0.95 \\
\hline & 1 & $10(14.7)$ & $3(11.1)$ & 1 & & 1 & \\
\hline & 2 & $10(14.7)$ & $5(18.5)$ & $0.436(0.056-3.418)$ & & $1.667(0.311-8.929)$ & \\
\hline & 3 & $14(20.6)$ & $5(18.5)$ & $0.388(0.069-2.163)$ & & $1.190(0.230-6.170)$ & \\
\hline & 4 & $34(50.0)$ & $14(51.9)$ & $0.477(0.084-2.698)$ & & $1.373(0.328-5.750)$ & \\
\hline \multirow[t]{3}{*}{ Paralysis } & & & & & $0.03^{\mathrm{a})}$ & & $0.02^{\text {a) }}$ \\
\hline & No & $46(67.6)$ & $11(40.7)$ & 1 & & 1 & \\
\hline & Yes & $22(32.4)$ & $16(59.3)$ & $6.380(1.256-32.409)$ & & $3.041(1.211-7.636)$ & \\
\hline \multirow[t]{5}{*}{ BMI $\left(\mathrm{kg} / \mathrm{m}^{2}\right)$} & & & & & 0.82 & & 0.70 \\
\hline & $<20$ & $19(27.9)$ & $10(37.0)$ & $2.790(0.026-294.553)$ & & $1.722(0.621-4.781)$ & \\
\hline & 20 to $<25$ & $36(52.9)$ & $11(40.7)$ & 1 & & 1 & \\
\hline & 25 to $<30$ & $9(13.2)$ & $4(14.8)$ & $3.050(0.076-121.812)$ & & $1.455(0.374-5.654)$ & \\
\hline & $\geq 30$ & $4(5.9)$ & $2(7.4)$ & $4.513(0.164-124.332)$ & & $1.636(0.263-10.168)$ & \\
\hline Weight, mean $\pm S D$ (kg) & & $61.21 \pm 1.74$ & $59.83 \pm 2.41$ & $1.021(0.936-1.114)$ & 0.64 & $1.025(0.983-1.068)$ & 0.66 \\
\hline \multirow[t]{3}{*}{ Diabetes } & & & & & 0.92 & & 0.84 \\
\hline & No & $49(72.1)$ & $20(74.1)$ & 1 & & 1 & \\
\hline & Yes & $19(27.9)$ & $7(25.9)$ & $1.083(0.215-5.466)$ & & $0.903(0.329-2.480)$ & \\
\hline \multirow[t]{3}{*}{ ESRD } & & & & & 1.00 & & 0.84 \\
\hline & № & $62(91.2)$ & $27(100)$ & 1 & & 1 & \\
\hline & Yes & $6(8.8)$ & 0 & - & & $0.903(0.329-2.480)$ & \\
\hline \multirow[t]{3}{*}{ Smoker } & & & & & 0.07 & & 0.38 \\
\hline & No & $58(85.3)$ & $21(77.8)$ & 1 & & 1 & \\
\hline & Yes & $10(14.7)$ & $6(22.2)$ & $4.348(0.883-21.402)$ & & $1.657(0.536-5.122)$ & \\
\hline \multirow[t]{3}{*}{ Culture } & & & & & 0.77 & & 0.28 \\
\hline & Negative & $37(54.4)$ & $18(66.7)$ & 1 & & 1 & \\
\hline & Positive & $31(45.6)$ & $9(33.3)$ & $0.800(0.179-3.579)$ & & $0.597(0.235-1.515)$ & \\
\hline \multirow[t]{4}{*}{ Site } & & & & & 0.26 & & 0.39 \\
\hline & Ischial area & $14(20.6)$ & $8(29.6)$ & 1 & & 1 & \\
\hline & Sacral area & $45(66.2)$ & $14(51.9)$ & $4.458(0.564-35.211)$ & & $0.544(0.189-1.565)$ & \\
\hline & Trochanter & $9(13.2)$ & $5(18.5)$ & $4.089(0.691-24.196)$ & & $0.972(0.241-3.928)$ & \\
\hline Area, mean $\pm S D\left(\mathrm{~cm}^{2}\right)$ & & $25.92 \pm 4.75$ & $37.08 \pm 6.27$ & $0.984(0.969-1.000)$ & 0.06 & $0.985(0.971-0.999)$ & $0.03^{\mathrm{a})}$ \\
\hline \multirow[t]{6}{*}{ Braden scale } & & & & & 0.54 & & 0.78 \\
\hline & Severe risk & $3(4.4)$ & $3(11.1)$ & 1 & & 1 & \\
\hline & High risk & $15(22.1)$ & $5(18.5)$ & $0.286(0.100-8.259)$ & & $0.333(0.050-2.214)$ & \\
\hline & Moderate risk & $25(36.8)$ & $8(29.6)$ & $1.078(0.062-18.886)$ & & $0.375(0.064-2.211)$ & \\
\hline & Mild risk & $21(30.9)$ & $7(25.9)$ & $2.055(0.160-26.324)$ & & $0.400(0.066-2.415)$ & \\
\hline & No risk & $4(5.9)$ & $4(14.8)$ & $0.796(0.058-10.850)$ & & $0.667(0.060-7.352)$ & \\
\hline \multirow[t]{4}{*}{ Albumin (g/dL) } & & & & & 0.33 & & 0.29 \\
\hline & $<3.0$ & $18(26.5)$ & $4(14.8)$ & $0.269(0.031-2.376)$ & & $0.563(0.163-1.940)$ & \\
\hline & 3.0 to $<3.5$ & $12(17.6)$ & $8(29.6)$ & $0.225(0.030-1.668)$ & & $1.689(0.576-4.953)$ & \\
\hline & 3.5 to $<5.2$ & $38(55.9)$ & $15(55.6)$ & 1 & & 1 & \\
\hline \multirow[t]{3}{*}{ Hemoglobin ( $\mathrm{g} / \mathrm{dL})$} & & & & & 0.24 & & 0.18 \\
\hline & $\geq 10.7$ & $43(63.2)$ & $13(48.1)$ & 1 & & 1 & \\
\hline & $<10.7$ & $25(36.8)$ & $14(51.9)$ & $1.834(0.664-5.063)$ & & $1.852(0.752-4.563)$ & \\
\hline
\end{tabular}

Odds ratio (OR) and $95 \%$ confidence intervals (Cls) computed in the subsample of flap complication; No vs. yes, P-value computed using Student t-test and Mann-Whitney U test in continuous variables; P-value computed using chi-square test and Fisher exact test in categorical variables, age, weight, and area were continuous variables and expressed mean \pm SD. ECOG, eastern cooperative oncology group; BMI, body mass index; ESRD, end-stage renal disease.

a) $\mathrm{P}<0.05$. 
Lee $\mathrm{D}$ et al.

Outcome of flap reconstruction of sore

Table 4. Analysis of effects of clinical variables on different site sore development

\begin{tabular}{|c|c|c|c|c|c|c|c|}
\hline \multirow{2}{*}{ Variable } & \multirow{2}{*}{ Subgroup } & \multicolumn{2}{|c|}{ Developing sore of other sites, №. (\%) } & \multicolumn{2}{|c|}{ Unadjusted analysis } & \multicolumn{2}{|c|}{ Adjusted analysis } \\
\hline & & No & Yes & OR (95\% Cls) & P-value & OR (95\% Cls) & P-value \\
\hline Age, mean $\pm S D$ (yr) & & $54.05 \pm 19.31$ & $52.71 \pm 13.31$ & $1.004(0.964-1.045)$ & 0.85 & $0.955(0.852-1.071)$ & 0.43 \\
\hline \multirow[t]{3}{*}{ Sex } & & & & & 0.46 & & 0.70 \\
\hline & Female & 38 (43.2) & $2(28.6)$ & 1 & & 1 & \\
\hline & Male & $50(56.8)$ & $5(71.4)$ & $1.900(0.349-10.330)$ & & $1.900(0.349-10.330)$ & \\
\hline \multirow[t]{5}{*}{ ECOG performance status } & & & & & 0.91 & & 0.84 \\
\hline & 1 & $13(14.8)$ & 0 & 1 & & 1 & \\
\hline & 2 & $14(15.9)$ & $1(14.3)$ & $1.914(0.262-14.000)$ & & $2.793(0.105-74.630)$ & \\
\hline & 3 & $18(20.5)$ & $1(14.3)$ & $1.628(0.175-15.141)$ & & $2.189(0.083-57.985)$ & \\
\hline & 4 & $43(48.9)$ & $5(71.4)$ & $2.093(0.228-19.202)$ & & $3.414(0.177-65.801)$ & \\
\hline \multirow[t]{3}{*}{ Paralysis } & & & & & $0.03^{\mathrm{a})}$ & & $0.02^{\mathrm{a})}$ \\
\hline & No & $56(63.6)$ & $1(14.3)$ & 1 & & 1 & \\
\hline & Yes & $32(36.4)$ & $6(85.7)$ & $10.500(1.210-91.147)$ & & $10.500(1.210-91.151)$ & \\
\hline \multirow[t]{5}{*}{$\mathrm{BMI}\left(\mathrm{kg} / \mathrm{m}^{2}\right)$} & & & & & 0.42 & & 0.85 \\
\hline & $<20$ & $26(29.5)$ & $3(42.9)$ & $1.053(0.164-6.776)$ & & $1.240(0.257-5.987)$ & \\
\hline & 20 to $<25$ & $43(48.9)$ & $4(57.1)$ & 1 & & 1 & \\
\hline & 25 to $<30$ & $13(14.8)$ & 0 & $0.611(0.098-3.797)$ & & $0.358(0.018-7.084)$ & \\
\hline & $\geq 30$ & $6(6.8)$ & 0 & $0.889(0.113-7.016)$ & & $0.744(0.036-15.480)$ & \\
\hline Weight (kg) & & $61.07 \pm 14.12$ & $57.57 \pm 7.50$ & $1.020(0.961-1.082)$ & 0.52 & $0.776(0.548-1.099)$ & 0.15 \\
\hline \multirow[t]{3}{*}{ Diabetes } & & & & & 0.70 & & 1 \\
\hline & No & $64(72.7)$ & $5(71.4)$ & 1 & & 1 & \\
\hline & Yes & $24(27.3)$ & $2(28.6)$ & $0.618(0.056-6.838)$ & & $1.067(0.194-5.872)$ & \\
\hline \multirow[t]{3}{*}{ ESRD } & & & & & - & & 1 \\
\hline & No & 82 (93.2) & $7(100)$ & 1 & & 1 & \\
\hline & Yes & $6(6.8)$ & 0 & - & & $0.846(0.043-16.527)$ & \\
\hline \multirow[t]{3}{*}{ Smoker } & & & & & 0.69 & & 1 \\
\hline & No & $73(83.0)$ & $6(85.7)$ & 1 & & 1 & \\
\hline & Yes & $15(17.0)$ & $1(14.3)$ & $0.427(0.007-26.650)$ & & $0.811(0.091-7.238)$ & \\
\hline \multirow[t]{3}{*}{ Culture } & & & & & 0.87 & & 0.70 \\
\hline & Negative & $50(56.8)$ & $5(71.4)$ & 1 & & 1 & \\
\hline & Positive & 38 (43.2) & $2(28.6)$ & $1.473(0.012-175.900)$ & & $0.526(0.097-2.862)$ & \\
\hline \multirow[t]{4}{*}{ Site } & & & & & 0.55 & & 0.53 \\
\hline & Ischial area & $21(23.9)$ & $1(14.3)$ & 1 & & 1 & \\
\hline & Sacral area & $55(62.5)$ & $4(57.1)$ & $2.292(0.376-13.984)$ & & $1.527(0.161-14.466)$ & \\
\hline & Trochanter & $12(13.6)$ & $2(28.6)$ & $3.500(0.286-42.769)$ & & $3.500(0.286-42.771)$ & \\
\hline Area $\left(\mathrm{cm}^{2}\right)$ & & $28.79 \pm 38.53$ & $32.86 \pm 24.20$ & $0.997(0.979-1.016)$ & 0.79 & $0.993(0.961-1.025)$ & 0.66 \\
\hline \multirow[t]{6}{*}{ Braden scale } & & & & & 0.66 & & 0.73 \\
\hline & Severe risk & $5(5.7)$ & $1(14.3)$ & 1 & & 1 & \\
\hline & High risk & $18(20.5)$ & $2(28.6)$ & $0.750(0.078-7.210)$ & & $0.556(0.041-7.457)$ & \\
\hline & Moderate risk & 31 (35.2) & $2(28.6)$ & $0.500(0.064-3.906)$ & & $0.500(0.043-5.813)$ & \\
\hline & Mild risk & $28(31.8)$ & 0 & $0.562(0.080-3.939)$ & & $0.185(0.010-3.473)$ & \\
\hline & No risk & $6(6.8)$ & $2(28.6)$ & $0.600(0.084-4.294)$ & & $0.333(0.011-10.108)$ & \\
\hline \multirow[t]{4}{*}{ Albumin (g/dL) } & & & & & 0.18 & & 0.43 \\
\hline & $<3.0$ & $21(23.9)$ & $1(14.3)$ & $0.021(0.000-1.340)$ & & $0.794(0.078-8.075)$ & \\
\hline & 3.0 to $<3.5$ & $17(19.3)$ & $3(42.9)$ & $0.165(0.090-2.691)$ & & $2.941(0.541-15.976)$ & \\
\hline & 3.5 to $<5.2$ & $50(56.8)$ & $3(42.9)$ & 1 & & 1 & \\
\hline \multirow[t]{3}{*}{ Hemoglobin (g/dL) } & & & & & $0.04^{\mathrm{a})}$ & & $0.02^{\mathrm{a})}$ \\
\hline & $\geq 10.7$ & $55(62.5)$ & $1(14.3)$ & 1 & & 1 & \\
\hline & $<10.7$ & $33(37.5)$ & $6(85.7)$ & $10.000(1.153-86.759)$ & & $10.000(1.153-86.762)$ & \\
\hline
\end{tabular}

Odds ratio (OR) and $95 \%$ confidence intervals (Cls) computed in the subsample of flap complication; No vs. yes, P-value computed using Student t-test and Mann-Whitney U test in continuous variables; P-value computed using chi-square test and Fisher exact test in categorical variables, age, weight, and area were continuous variables and expressed mean \pm SD. ECOG, eastern cooperative oncology group; BMI, body mass index; ESRD, end-stage renal disease.

a) $\mathrm{P}<0.05$. 


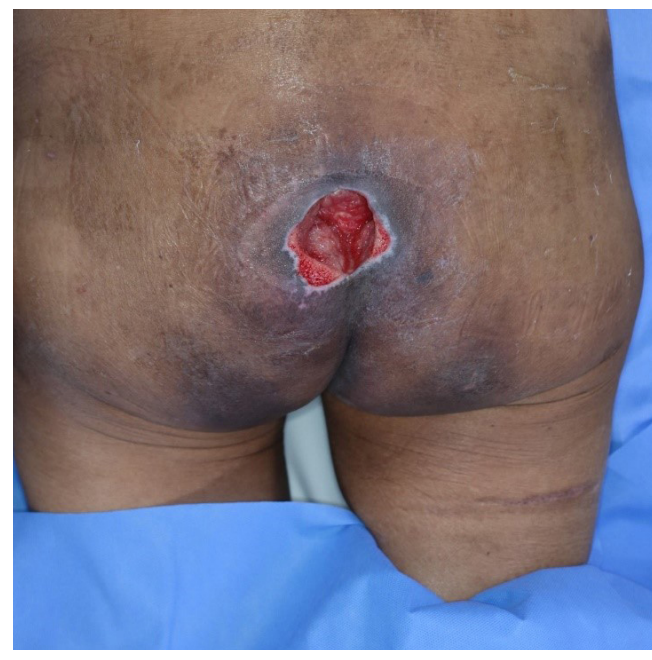

Fig. 1. Preoperative photograph of pressure sore. After debridement, soft tissue defect was about $4 \times 4 \mathrm{~cm}$.

min levels, hemoglobin levels, and weight were not significantly related.

The relationships between clinical variables and the need for reoperation (Table 3) or new ulcer occurrence (Table 4) are each presented in subsequent tables. Patients with paralysis had a significant likelihood of reoperation and new pressure ulcer occurrence at other locations. In the adjusted model, patients with a larger wound tended to undergo reoperation. Other variables did not show any significant results. Relapse did not show any significant correlation with any variable.

\section{Discussion}

Open wounds from pressure ulcers cause various problems for patients. Pressure ulcers decrease the quality of life and can cause wound site infection, leading to increased mortality and length of hospital stay [14]. For example, Espejo et al. [14] demonstrated that there was a correlation between compression ulcers and bacteremia associated with mortality using a prospective study design.

Many plastic surgeons are aware of these problems and have explored how to optimally cover open wounds caused by pressure ulcers. Patients with severe pressure ulcers that fail to heal require surgery to fill the wounds and prevent any further tissue damage. A variety of reconstructive options are available, such as split thickness skin grafting, local flaps, regional flaps, and microvascular free flaps [15]. With improved understanding of vascularity, flap surgery has become popular, because it can provide rich soft tissue and is therefore more favorable

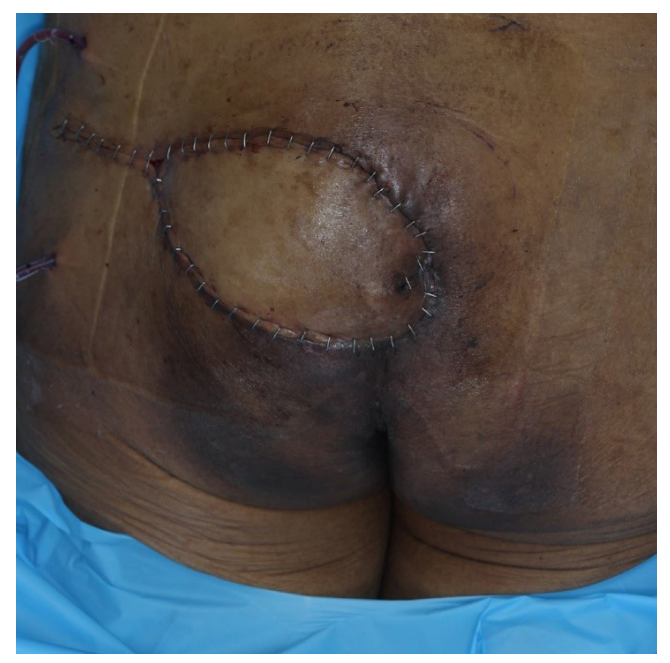

Fig. 2. Immediate postoperative photograph of pressure sore. The defect was covered by superior gluteal artery based fasciocutaneous flap.

than primary closure of skin graft for covering pressure sore wounds (Figs. 1, 2). However, complication rates after flap coverage make it difficult to determine which treatment option is most suitable.

The possibility of postoperative complications after flap surgery turned out to vary widely among different studies. In the study of Bamba et al. [11], the probability of flap complication after coverage was $52 \%$. Other studies reported the incidence of complications to be $21 \%, 29 \%, 82 \%$, and $39 \%$.

In this study, the larger the defect size created after the last debridement operation before the flap surgery, the higher the probability of flap complications and need for reoperation. The larger the defect, the larger a flap was required to cover the wound. Although the wound size has not been specifically indicated as a risk factor in pressure sore reconstruction, it has already been identified as an important risk factor in flap reconstruction [16]. In addition, large wounds are inherently more prone to infection and wound dehiscence; therefore, the risk of reoperation is high in these cases.

Flap complications were also more common in smokers. Compared with nonsmokers, smokers are known to have worse microcirculation and more frequent complications of flap surgery including flap necrosis and failure [17]. Among the risk factors identified in this study, smoking is the only one that patients can modify. Therefore, surgeons should induce patients to quit smoking before the surgery by recommending appropriate smoking cessation programs.

The probability of complications was high when bacteria was identified in the wound culture before surgery. Patients 
with a positive intraoperative culture are known to have an increased rate of infection $[18,19]$. In general, patients who are indicated for surgery to cover a pressure sore are those who do not have any acute medical problems, with no purulent infection at the wound site and well-formed granulation tissue [20]. We also performed surgery on patients whose wounds were relatively clean and well-managed without pus on evaluation. While no patient in this study had purulent wounds, $42.1 \%$ showed positive culture swab tests on the first day of hospitalization. Therefore, using appropriate antibiotics according to the culture test results may help reduce postoperative complications.

We considered factors such as BMI, albumin, and hemoglobin to assess the nutritional status of patients. Generally, severe malnutrition can be a risk factor for the development of pressure ulcers [21]. However, no correlation was found between nutritional factors and probability of complications in this study. Among the many ways to assess nutritional status, in this study we used the conventional indicators mentioned above. However, because these indicators are controversial in terms of sensitivity and specificity [22], further research using criteria that better reflect malnutrition is needed.

The limitations of this study mainly owe to its retrospective nature. Furthermore, outcomes such as reoperation were influenced by preference of the surgeons. Also, we focused on complications described in the medical records, meaning that complications that are easily overlooked may have been omitted. Further, according to the protocol in our department, we routinely perform a culture test on the first day of hospitalization. However, in one third of patients, intraoperative culture tests were not performed. Considering the rate of false positives in swab tests in wound cultures, a strong correlation between infection and complications could have been revealed if an intraoperative culture test were performed. It is also unfortunate that there was insufficient long-term follow-up because of the characteristics of patients with pressure ulcers, such as difficulty in accessing the hospital, or instances of those who died at home or other institutions before the follow-up. Although we found significant results from several factors, it is hard to determine the impact of confounding variables such as types of flaps, composition, method of movement, and proximity to defect.

Only a limited number of patients with pressure sores were eligible for flap surgery. Most patients with pressure ulcers already had general deterioration or spinal cord injury, increasing the probability of postoperative recurrence and making complication management difficult.

This study also confirmed that patients with paralysis were likely to need reoperation or suffer pressure ulcers in other areas. The size of the defect area also significantly increased the risk of flap complication and reoperation. Close monitoring should be performed in patients with paralysis or large defect areas. We revealed that smoking and infection increase the incidence of flap complications. Smoking cessation and adequate infection control will help reduce these risks. This study analyzed the factors affecting flap operation on pressure ulcers, aiming to contribute to more successful outcomes by raising awareness of significant factors before operation.

Complications after surgery for covering pressure ulcers are considered important because they can slow down wound recovery and put the donor site at risk. In this study, we investigated the factors influencing outcomes after flap surgery on an area damaged by a pressure ulcer. These results can be helpful in identifying patients prone to postoperative complications and in establishing surgical strategies to address risks.

\section{Conflict of interest}

No potential conflict of interest relevant to this article was reported.

\section{ORCID iDs}

Donghwan Lee https://orcid.org/0000-0002-5436-7911

Min Ji Kim

https://orcid.org/0000-0002-0723-4382

Hyung Min Hahn

https://orcid.org/0000-0002-4756-1804

\section{References}

1. Graves N, Birrell F, Whitby M. Effect of pressure ulcers on length of hospital stay. Infect Control Hosp Epidemiol 2005;26:293-7.

2. Bennett G, Dealey C, Posnett J. The cost of pressure ulcers in the UK. Age Ageing 2004;33:230-5.

3. Garibaldi RA, Brodine S, Matsumiya S. Infections among patients in nursing homes: policies, prevalence, problems. N Engl J Med 1981;305:731-5.

4. Scheckler WE, Peterson PJ. Infections and infection control among residents of eight rural Wisconsin nursing homes. Arch Intern Med 1986;146:1981-4.

5. Disa JJ, Carlton JM, Goldberg NH. Efficacy of operative cure in pressure sore patients. Plast Reconstr Surg 1992;89: 
272-8.

6. Thiessen FE, Andrades P, Blondeel PN, et al. Flap surgery for pressure sores: should the underlying muscle be transferred or not? J Plast Reconstr Aesthet Surg 2011;64:84-90.

7. Schryvers OI, Stranc MF, Nance PW. Surgical treatment of pressure ulcers: 20-year experience. Arch Phys Med Rehabil 2000;81:1556-62.

8. Sameem M, Au M, Wood T, et al. A systematic review of complication and recurrence rates of musculocutaneous, fasciocutaneous, and perforator-based flaps for treatment of pressure sores. Plast Reconstr Surg 2012;130:67e-77e.

9. Eriksson E, Hietanen H, Asko-Seljavaara S. Prevalence and characteristics of pressure ulcers: a one-day patient population in a Finnish city. Clin Nurse Spec 2000;14:119-25.

10. Biglari B, Buchler A, Reitzel T, et al. A retrospective study on flap complications after pressure ulcer surgery in spinal cord-injured patients. Spinal Cord 2014;52:80-3.

11. Bamba R, Madden JJ, Hoffman AN, et al. Flap reconstruction for pressure ulcers: an outcomes analysis. Plast Reconstr Surg Glob Open 2017;5:e1187.

12. Keys KA, Daniali LN, Warner KJ, et al. Multivariate predictors of failure after flap coverage of pressure ulcers. Plast Reconstr Surg 2010;125:1725-34.

13. Lindqvist EK, Sommar P, Stenius M, et al. Complications after pressure ulcer surgery: a study of 118 operations in spinal cord injured patients. J Plast Surg Hand Surg 2020; 54:145-50.
14. Espejo E, Andres M, Borrallo RM, et al. Bacteremia associated with pressure ulcers: a prospective cohort study. Eur J Clin Microbiol Infect Dis 2018;37:969-75.

15. Bhattacharya S, Mishra RK. Pressure ulcers: current understanding and newer modalities of treatment. Indian J Plast Surg 2015;48:4-16.

16. Shea P, O'Hara NN, Sprague SA, et al. Wound surface area as a risk factor for flap complications among patients with open fractures. Plast Reconstr Surg 2018;142:228-36.

17. Reus WF, Robson MC, Zachary L, et al. Acute effects of tobacco smoking on blood flow in the cutaneous micro-circulation. Br J Plast Surg 1984;37:213-5.

18. Edwards R, Harding KG. Bacteria and wound healing. Curr Opin Infect Dis 2004;17:91-6.

19. Garibaldi RA, Cushing D, Lerer T. Risk factors for postoperative infection. Am J Med 1991;91:158S-163S.

20. Boyko TV, Longaker MT, Yang GP. Review of the current management of pressure ulcers. Adv Wound Care (New Rochelle) 2018;7:57-67.

21. Pinchcofsky-Devin GD, Kaminski MV Jr. Correlation of pressure sores and nutritional status. J Am Geriatr Soc 1986;34:435-40.

22. Zhang Z, Pereira SL, Luo M, et al. Evaluation of blood biomarkers associated with risk of malnutrition in older adults: a systematic review and meta-analysis. Nutrients 2017;9:829. 